\title{
THE SECONDARY CHARACTERISTIC CLASSES OF SOLVABLE FOLIATIONS
}

\author{
WILLIAM T. PELLETIER
}

\begin{abstract}
The secondary characteristic classes of a codimension $q$ foliation of a manifold $M$ are certain cohomology classes of $M$ which are constructed from the curvature matrix of a torsion-free connection on the normal bundle of the foliation. We consider the foliation of a real or complex Lie group $G$ by the left cosets of a closed connected subgroup $H$ and any foliation obtained from it through dividing by a discrete subgroup $\Gamma \subset G$. Such foliations are homogeneous. The Fuks-Pittie conjecture for homogeneous foliations is that the secondary classes are generated by $\left\{h_{1} h_{i 2} \cdots h_{i} \tau_{1}^{q}\right\}$. We prove the Fuks-Pittie conjecture if $H$ is reductive or solvable. We also prove an Addition Theorem for the classes which can be applied to reduce the general problem of calculating the secondary classes to the case in which $G$ is semisimple.
\end{abstract}

A natural type of foliation to study, denoted $(G, H)$, is that of a connected Lie group $G$ foliated by the left cosets of a closed connected subgroup $H$. Similarly, there is a holomorphic foliation $\left(G^{c}, H^{c}\right)$ of complex Lie groups corresponding to the complexified Lie algebras $\mathfrak{g} \otimes \mathbf{C}$ and $\mathfrak{h} \otimes \mathbf{C}$. Another related foliation is obtained from $(G, H)$ through dividing by a discrete subgroup $\Gamma \subset G$ acting on the left of $G$ to obtain a foliation of $\Gamma \backslash G$ with leaves diffeomorphic to $H \cap \Gamma \backslash H$. These three types of Lie group foliations are called homogeneous. The secondary classes are de Rham cohomology classes of $G, G^{c}$ or $\Gamma \backslash G$; they are foliation invariants constructed from a torsion-free connection and its curvature on the normal bundle of the foliation. A special homogeneous connection is used which facilitates the reduction of the calculation of the foliation classes to the Lie algebra level; as a result, the classes for all three types of homogeneous foliations $(\mathfrak{g}, \mathfrak{h})$ can then be computed simultaneously in the complex of left-invariant complex-valued differential forms $\Lambda^{*}(g \otimes \mathbf{C}) *$

The general problem with which this paper is concerned is the calculation of these classes for codimension $q$ homogeneous foliations. Kamber and Tondeur [5] have proved some results similar to those of this paper using different methods. But their secondary classes are not the same as the Bott-Haefliger classes studied here; although constructed analogously, their classes do not come from the cohomology of $B \Gamma_{q}$. Bott and Haefliger [2] have shown that the stable classes $\left(i_{1}+|J|>q+1\right.$; see $\S 1$ for notation) vanish for (semisimple, parabolic) homogeneous foliations. A

Received by the editors September 20, 1982.

1980 Mathematics Subject Classification. Primary 53C12, 57R20, 57R30.

Key words and phrases. Foliation, homogeneous foliation, connection, curvature, Lie algebra, secondary characteristic class. 
systematic approach to the general problem using Lie algebras has been developed by Pittie [6]; he applied it to (semisimple, parabolic) homogeneous foliations and showed that all the nonzero classes are moveable and are of the form $h_{1} h_{i_{2}} \cdots h_{i_{h}} \tau_{1}^{q}$. My interest in this problem was stimulated by the Fuks-Pittie conjecture that the homogeneous foliation classes are generated by $\left\{h_{1} h_{i_{2}} \cdots h_{i_{h}} \tau_{1}^{q}\right\}$. Fuks [3] had conjectured that the classes are essentially the same as those for the specific case $(S l(n), P)$ where $P \subset S l(n)$ is the maximal parabolic subgroup such that $S l(n) / P \cong$ $\mathbf{P}^{n^{-1}}$. This is not quite true as Pittie [6] has explained and as Baker's [1] examples show. But Pittie's modified version of the conjecture appears to be true; he has proved it for parabolic foliations, and the results of this paper verify it for solvable foliations.

THEOREM 5. The only secondary characteristic class of a solvable homogeneous foliation which can be nonzero is the Godbillon-Vey class, $h_{1} \tau_{1}^{q}$.

The key ingredient in the proof of Theorem 5 is the following basic structure theorem for the secondary classes of homogeneous foliations $(\mathfrak{g}, \mathfrak{h})$.

ThEOREM 2 (AdDition TheOREM). The Levi decomposition $0 \rightarrow \Re \rightarrow \mathfrak{g} \stackrel{\pi}{\rightarrow} \mathfrak{g} \rightarrow 0$ of $\mathfrak{g}=\mathfrak{g} \ltimes \mathcal{R}$ induces homogeneous foliations $(\mathcal{R}, \mathfrak{g} \cap \mathcal{R})$ and $(\mathfrak{g}, \pi(\mathfrak{h}))$ with addition formulas

$$
\tau_{i}(\mathfrak{g})=\tau_{i}(\mathfrak{g})+\tau_{i}(\Re) \text { and } h_{i}(\mathfrak{g})=h_{i}(\mathfrak{g})+h_{i}(\Re) .
$$

The significance of the Addition Theorem is that it facilitated the reduction of the ( $g$, solvable) case of Theorem 5 to component foliations of the types (solvable, solvable) and (semisimple, solvable) for which the secondary classes were calculated separately (Theorems 3 and 4) and then combined to prove Theorem 5. Moreover, the significance of the Addition Theorem extends beyond its application to solvable foliations. The author has used it in combination with the solvable result to reduce the case of an arbitrary homogeneous foliation $(\mathfrak{g}, \mathfrak{h})$ to the case where $\mathfrak{g}$ is semisimple. This argument will be given in a subsequent paper where the Fuks-Pittie conjecture is verified for (semisimple, maximal rank) homogeneous foliations; those computations are of an entirely different type from the ones for the solvable case presented here. Thus in order to complete the proof of the Fuks-Pittie conjecture it remains only to calculate the secondary classes for (semisimple, nonmaximal rank) homogeneous foliations. Based on many detailed computational examples, the author conjectures that such classes all vanish; yet it is presently an open question.

It is a pleasure to thank Harsh Pittie for suggesting this problem to me and for many illuminating conversations throughout the course of this work.

1. Secondary characteristic classes of homogeneous foliations. In this section we establish notation, briefly review the construction of the secondary classes, and explain Lie algebra techniques which greatly simplify calculation of the homogeneous foliation classes.

Let $M$ be a foliated manifold of codimension $q$ with $E \subset T M$ the involutive subbundle tangent to the foliation and $Q \equiv T M / E$ the normal bundle. Recall that a 
Bott connection (also called basic or torsion free) is a connection $\nabla: \Gamma(T M) \rightarrow$ $\operatorname{Hom}(\Gamma(Q), \Gamma(Q))$ on $Q$ which is "adapted" to the foliation in the sense that $\nabla$ is a Lie algebra homomorphism when restricted to $\Gamma(E)$. Comparison of a Bott connection on $Q$ with a flat connection (homogeneous foliations have flat normal bundles) yields the foliation classes as follows. Let $\theta_{1}$ and $\Omega_{1} \equiv d \theta_{1}-\theta_{1} \wedge \theta_{1}$ be the connection and curvature matrices for a Bott connection on $Q$ and let $\theta_{0}$ and $\Omega_{0}=0$ be those for a flat connection; $\theta_{t} \equiv t \theta_{1}+(1-t) \theta_{0}$ is a connection on the pull-back bundle $\pi^{*} Q \rightarrow M \times[0,1]$ with curvature $\Omega_{t}$. Then an additive basis for the foliation classes is given by the de Rham cohomology classes of $M$ represented by the differential forms $h_{I} \tau_{J} \equiv h_{i_{1}} \cdots h_{i_{h} j_{1}} \tau_{j_{j_{l}}}$ subject to the Vey conditions (Godbillon [4])

(1) $i_{1}<\cdots<i_{k}, j_{1} \leqslant \cdots \leqslant j_{l}, i_{1} \leqslant j_{1}$

(2) $i_{1}+|J| \geqslant q+1$ where $|J|=j_{1}+\cdots+j_{l}$,

(3) $|J| \leqslant q$,

where $\tau_{j}=\operatorname{Tr}\left(\Omega_{1}^{j}\right), h_{j}=\int_{0}^{l} \operatorname{Tr}\left(\Omega_{t}^{j}\right)$, and consequently $d h_{j}=\tau_{j}$ (Chern-Weil theory).

The calculation of the foliation classes will actually be done using a Bott connection on $Q^{*}$ rather than on $Q$. The reason for this is that a local Bott connection matrix $\left(\theta_{i j}\right)$ for $Q^{*}$ can be easily computed by differentiating the local basis l-forms $\left\{\omega_{i}\right\}$ for the differential ideal $Q^{*}: d \omega_{i}=\sum_{j=1}^{q} \theta_{i j} \wedge \omega_{j}$. This will have no effect on the $h$ 's and $\tau$ 's other than a sign change for the odd $\tau$ 's and corresponding $h$ 's.

Now for a homogeneous foliation $(G, H)$ there are left-invariant trivializations $T G \simeq G \times \mathfrak{g}, E \simeq G \times \mathfrak{h}$, and $Q \simeq G \times \mathfrak{g} / \mathfrak{h}$ where $\mathfrak{g}$ and $\mathfrak{h}$ denote the Lie algebras of $G$ and $H$. As a result a connection on $Q$ is determined by a real linear map $\nabla$ : $\mathfrak{g} \times \mathfrak{g} / \mathfrak{h} \rightarrow \Gamma(Q)$. In order for $\nabla$ to reflect the homogeneity of the foliation, $\nabla$ is required to be left-invariant, and hence a left-invariant connection on $Q$ is specified by a real linear map $\nabla: \mathfrak{g} \times \mathfrak{g} / \mathfrak{h} \rightarrow \mathfrak{g} / \mathfrak{h}$. A left-invariant connection is homogeneous if it agrees with the adjoint action of $\mathfrak{h}$ on $\mathfrak{g} / \mathfrak{h}$. Such a connection is then automatically a Bott connection since the adjoint action is a Lie algebra homomorphism. One method of constructing a homogeneous connection is to choose a splitting $\sigma: \mathfrak{g} / \mathfrak{h} \rightarrow \mathfrak{g}$ of $0 \rightarrow \mathfrak{h} \rightarrow \mathfrak{g} \stackrel{\pi}{\rightarrow} \mathfrak{g} / \mathfrak{h} \rightarrow 0$ and to define $\nabla_{X} Y=\pi[X, \sigma(Y)]$. The foliation classes are independent of the particular choice of homogeneous connection by (integrable) homotopy invariance of cohomology.

At this point it is important to note that the calculation of the secondary classes for homogeneous foliations may now be done at the Lie algebra level. Since the entries of the matrix of a homogeneous connection $\nabla: \mathfrak{g} \rightarrow \operatorname{Hom}(\mathfrak{g} / \mathfrak{h}, \mathfrak{g} / \mathfrak{h})$ are clearly in $\mathrm{g}^{*}$, the curvature and hence the secondary forms may be computed by working solely in the complex $\Lambda^{*} \mathrm{~g}^{*}$ of left-invariant differential forms. A particular type of homogeneous connection will be used to simplify these computations; it was first introduced by Pittie [6]. A special connection $\nabla: \mathfrak{g} \times \mathfrak{g} / \mathfrak{h} \rightarrow \mathfrak{g} / \mathfrak{h}$ is defined by

$$
\nabla_{X} Y=\pi[X-\sigma \pi(X), \sigma(Y)]
$$

for some splitting $\sigma: \mathfrak{g} / \mathfrak{h} \rightarrow \mathfrak{g}$. The virtue of the special connection is that it captures only the adjoint action of $\mathfrak{h}$ on $\mathfrak{g} / \mathfrak{h}$ and entirely ignores the bracket relations in 
$\sigma(\mathfrak{g} / \mathfrak{h})$. Thus the connection and curvature matrices are far more tractable than those for an arbitrary homogeneous connection; without the special connection the Lie algebra calculations would be prohibitively arduous.

Now let $\theta$ be a special connection matrix with respect to some basis of $\mathfrak{g} / \mathfrak{h}$. By construction $\theta_{i j} \in \mathfrak{h}^{*} ; \theta$ is the matrix of the adjoint representation of $\mathfrak{h}$ on $\mathfrak{g} / \mathfrak{h}$. Explicitly, $\theta$ may be computed by differentiating the dual basis elements of $(\mathfrak{g} / \mathfrak{h})^{*}$, reading off the coefficients of the basis elements, and projecting them to $\mathfrak{h}^{*}$. The associated special curvature matrix $\Omega=d \theta-\theta \wedge \theta$ may be computed by differentiating $\theta$ and then projecting to $\mathfrak{h}^{*} \wedge(\mathfrak{g} / \mathfrak{h})^{*}+\Lambda^{2}(\mathfrak{g} / \mathfrak{h})^{*}$; that is, $\Omega=d^{\prime \prime} \theta$ where $d^{\prime \prime}$ denotes the projection composed with the exterior derivative. This is a consequence of the special connection being a Lie algebra homomorphism on $\mathfrak{h}$ so that $\Omega$ vanishes on $\Lambda^{2} \mathfrak{h}$.

These computational techniques also apply to the secondary classes for the other two types of homogeneous foliations mentioned earlier; since the work will be done at the Lie algebra level, any homogeneous foliation will be denoted $(\mathfrak{g}, \mathfrak{h})$. In fact, the work is actually done in the complex case $(\mathfrak{g} \otimes \mathbf{C}, \mathfrak{h} \otimes \mathbf{C})$ in order to apply the structure theory of complex Lie algebras; the specific reason for working over $\mathbf{C}$ instead of over $\mathbf{R}$ is that it is only over $\mathbf{C}$ that Lie's theorem for solvable algebras applies and that a semisimple algebra is guaranteed a decomposition into 1-dimensional root spaces. The only difference is that if we start with a real foliation, then the foliation classes will lie in the complex cohomology ring instead of the real one. Thus attention will be restricted to complex Lie algebras pairs $(\mathfrak{g}, \mathfrak{h})$ in order to compute the secondary classes of homogeneous foliations.

2. Solvable foliations. The secondary characteristic classes of homogeneous foliations $(\mathfrak{g}, \mathfrak{h})$ with $\mathfrak{h}$ reductive or solvable are computed in this section. The reductive case (Theorem 1) is not difficult, but it illustrates some of the techniques used later on. For the solvable case, the Levi decomposition of $g$ is used first to prove a general Addition Theorem (Theorem 2) for the $h$ 's and $\tau$ 's of an arbitrary $(\mathfrak{g}, \mathfrak{h})$. The application of the Addition Theorem to a ( $g$, solvable) foliation enables me to compute the ( $\mathrm{g}$, solvable) classes in terms of the secondary classes for homogeneous foliations of the types (solvable, solvable) and (semisimple, solvable). Theorems 3 and 4 calculate the foliation classes for these two cases. Theorem 5 combines these two cases through the Addition Theorem to obtain the classes for ( $g$, solvable) foliations.

THEOREM 1. Let $\mathfrak{r}$ be a codim $q$ reductive subalgebra of a Lie algebra $\mathfrak{g}$. Then the foliation classes for the homogeneous foliation $(\mathrm{g}, \mathrm{r})$ vanish.

Proof. Since $r$ is reductive there exists a vector-space splitting $\sigma: \mathfrak{g} / \mathfrak{r} \rightarrow \mathfrak{g}$ of $0 \rightarrow \mathfrak{r} \rightarrow \mathfrak{g} \rightarrow \mathfrak{g} / \mathfrak{r} \rightarrow 0$ such that $V=\sigma(\mathfrak{g} / \mathrm{r})$ is an $\mathrm{r}$-module.

Let $\theta$ be the special connection matrix. Then the elements of the curvature matrix $\Omega=d^{\prime \prime} \theta$ lie in $\mathrm{r}^{*} \wedge V^{*}+V^{*} \wedge V^{*}$. Since $V$ is an $\mathfrak{r}$-module and $\theta_{i j} \in \mathrm{r}^{*}$, the $\mathrm{r}^{*} \wedge V^{*}$ component of $\Omega$ must vanish. Hence $\Omega_{i j} \in V^{*} \wedge V^{*}$. Thus if $f$ is an invariant polynomial of degree greater than $q / 2, f(\Omega)$ has degree greater than $q$. This means that $f(\Omega)=0$ since $\operatorname{dim} V=q$. Therefore $\tau_{J}=0$ for $|J|>q / 2$. 
Thus the only remaining classes are $h_{I} \tau_{J}$ with $|J| \leqslant q / 2$. But the condition $|J| \leqslant q / 2$ together with the conditions on the indices of the foliation classes (see §1) lead to a contradiction:

$$
i_{1}+|J| \geqslant q+1 \Rightarrow i_{1}+q / 2 \geqslant q+1 \Rightarrow i_{1}>q / 2+1,
$$

and then $j_{1} \geqslant i_{1} \Rightarrow j_{1}>q / 2+1$ which contradicts $|J| \leqslant q / 2$.

So the foliation classes must all vanish for foliations $(g, r)$ with $r$ reductive.

THEOREM 2 (AdDITION THEOREM). Let $(\mathfrak{g}, \mathfrak{h})$ be an arbitrary homogeneous foliation. The Levi decomposition of $\mathfrak{g}$ induces foliations $(\mathcal{R}(\mathfrak{g}), \mathfrak{h})$ and $\left(\mathfrak{g}(\mathfrak{g}), \mathfrak{h}^{\prime \prime}\right)$ with addition formulas $\tau_{i}(\mathrm{~g})=\tau_{i}(\Re)+\tau_{i}(\mathfrak{g})$ and $h_{i}(\mathrm{~g})=h_{i}(\Re)+h_{i}(\mathfrak{g})$.

Proof. Let $\mathcal{R}(\mathfrak{g}) \equiv \operatorname{Rad} \mathfrak{g}$ and $\mathfrak{s}(\mathfrak{g}) \equiv \mathfrak{g} /$ Rad $\mathfrak{g}$. Then using the Levi decomposition of $\mathfrak{g}$ there is a split exact sequence of Lie algebras $0 \rightarrow \mathcal{R}(\mathfrak{g}) \rightarrow \mathfrak{g} \stackrel{\pi}{\rightarrow} \mathfrak{g}(\mathfrak{g}) \rightarrow 0$ with $\mathfrak{g} \approx \mathfrak{s}(\mathfrak{g}) \ltimes \Re(g)$.

Define $\mathfrak{h}^{\prime}=\mathfrak{h} \cap \Re$ and $\mathfrak{h}^{\prime \prime}=\pi(\mathfrak{h})$. Thus there are foliations $\left(\Re, \mathfrak{h}^{\prime}\right)$ and $\left(\mathfrak{b}, \mathfrak{h}^{\prime \prime}\right)$. The goal is to extract information about the foliation classes for $(\mathfrak{g}, \mathfrak{h})$ from the foliations $\left(\Re, \mathfrak{h}^{\prime}\right)$ and $\left(\mathfrak{g}, \mathfrak{h}^{\prime \prime}\right)$.

Consider the following vector space decompositions: $\mathfrak{g}=\mathfrak{g}+\mathfrak{R}, \mathfrak{h}=\mathfrak{h}^{\prime \prime}+\mathfrak{h}^{\prime}$, and $(\mathfrak{g} / \mathfrak{h})^{*}=\left(\mathfrak{g} / \mathfrak{h}^{\prime \prime}\right)^{*}+\left(\mathfrak{R} / \mathfrak{h}^{\prime}\right)^{*}$. To compute the special connection matrix $\theta$ for $(\mathfrak{g}, \mathfrak{h})$, basis elements of $\left(\mathfrak{g} / \mathfrak{h}^{\prime \prime}\right)^{*}$ and $\left(\mathfrak{R} / \mathfrak{h}^{\prime}\right)^{*}$ are first differentiated (in $\left.\mathfrak{g}^{*}\right)$ and are then projected to $\mathfrak{h}^{*}$.

So first let $\omega \in\left(\mathfrak{s} / \mathfrak{h}^{\prime \prime}\right)^{*}$. Then

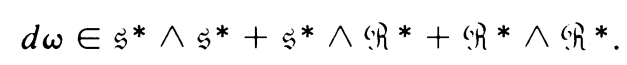

Since $6 R$ is an ideal and $d \omega()=,-\omega\left[\right.$, ], we must have $d \omega \in \mathfrak{F}^{*} \wedge \mathfrak{S}^{*}$. Thus the connection matrix has the form

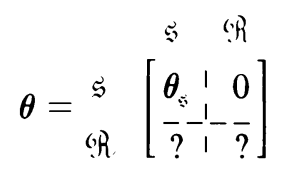

Now since $\mathfrak{s}$ is a subalgebra of $\mathfrak{g}=\mathfrak{s} \ltimes \mathcal{G}$ and $\mathscr{Q} R$ is an ideal, the exterior derivatives of $\mathfrak{g}$ and $\mathfrak{s}$ agree on $\mathfrak{s}^{*}$; that is, $d \omega=d_{\mathfrak{s}} \omega$. Therefore $\theta_{\mathfrak{s}}$ is the special connection matrix for the foliation $\left(\mathfrak{g}, \mathfrak{h}^{\prime \prime}\right)$.

Now let $\omega \in\left(\Re / \mathfrak{h}^{\prime}\right)^{*}$. Since $\mathfrak{S}$ is a subalgebra, $d \omega \in \mathfrak{S}^{*} \wedge \Re^{*}+\Re^{*} \wedge \Re^{*}$; so the ( $R, \Re)$ block of $\theta$ has entries in $\Re *$. By construction of the special connection the entries lie in $\mathfrak{h}^{*}$. Hence the entries lie in $\left(\mathfrak{h}^{\prime}\right)^{*}$. Also the $\Re^{*} \wedge \Re^{*}$ component of $d \omega$ is simply $d_{\Re} \omega$ (exterior derivative in $\Lambda^{*}\left(\Re *\right.$ rather than $\left.\Lambda^{*} g^{*}\right)$. Thus the ( $R, \Omega$ ) block of $\theta$ is simply the special connection matrix $\theta_{\Re}$ for the foliation $\left(\Re, \mathfrak{h}^{\prime}\right)$.

Thus the special connection matrix $\theta$ for $(\mathfrak{g}, \mathfrak{h})$ has the form

$$
\begin{aligned}
& \text { 马 } R
\end{aligned}
$$

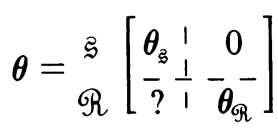


where $\theta_{\mathfrak{s}}$ and $\theta_{\mathscr{C}}$ are the respective special connection matrices for the foliations $\left(\mathfrak{g}, \mathfrak{h}^{\prime \prime}\right)$ and $\left(\mathfrak{R}, \mathfrak{h}^{\prime}\right)$. Then the respective curvature matrices $\Omega, \Omega_{\mathfrak{\xi}}$, and $\Omega_{\mathfrak{G}}$ are related by

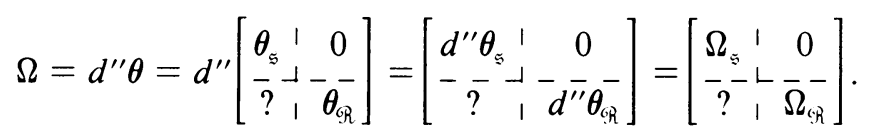

Now it is clear that the individual $h$ and $\tau$ forms for the three foliations satisfy the equations of the theorem.

THEOREM 3. Let $\mathfrak{h} \subset \mathfrak{g}$ be solvable Lie algebras. Then for the homogeneous foliation $(\mathfrak{g}, \mathfrak{h}), h_{i}=0$ for all $i>1$ and $\tau_{j}=0$ for all $j$.

Proof. Let $\mathfrak{n}=[\mathfrak{g}, \mathfrak{g}]$. Since $\mathfrak{g}$ is solvable, Lie's and Engel's theorems imply that $\mathfrak{n}$ is nilpotent, and $\mathfrak{a} \equiv \mathfrak{g} / \mathfrak{n}$ has positive dimension. Then there are exact sequences of Lie algebras $0 \rightarrow \mathfrak{n} \rightarrow \mathfrak{g} \stackrel{\pi}{\rightarrow} \mathfrak{a} \rightarrow 0$ and $0 \rightarrow \mathfrak{h}^{\prime} \rightarrow \mathfrak{h} \stackrel{\pi}{\rightarrow} \mathfrak{h}^{\prime \prime} \rightarrow 0$ where $\mathfrak{h}^{\prime}=\mathfrak{h} \cap \mathfrak{n}$ and $\mathfrak{h}^{\prime \prime}=\pi(\mathfrak{h})$, and there is an exact sequence of $\operatorname{ad}(\mathfrak{h})$-modules $0 \rightarrow \mathfrak{n} / \mathfrak{h}^{\prime} \rightarrow \mathfrak{g} / \mathfrak{h} \rightarrow$ $\mathfrak{a} / \mathfrak{h}^{\prime \prime} \rightarrow 0$.

The special connection matrix $\theta$ records the adjoint action $\mathfrak{h} \times \mathfrak{g} / \mathfrak{h} \rightarrow \mathfrak{g} / \mathfrak{h}$. To compute $\theta$, first choose a basis $\left\{v_{1}, \ldots, v_{n}\right\}$ of $n / h^{\prime}$ with respect to which the adjoint action of $\mathfrak{h}$ on the $\operatorname{ad}(\mathfrak{h})$-module $\mathfrak{n} / \mathfrak{h}^{\prime}$ is given by an upper triangular matrix. This is possible by Lie's Theorem because $\mathfrak{h}$ is solvable. Now extend $\left\{v_{1}, \ldots, v_{n}\right\}$ to get a basis $\left\{v_{1}, \ldots, v_{n}, w_{1}, \ldots, w_{m}\right\}$ for $\mathfrak{g} / \mathfrak{h}$. With respect to this basis, $\theta$ already has the form

$$
\theta={ }_{w}^{v}\left[\begin{array}{ccc}
v & w \\
\beta & 1 & ? \\
\hdashline & 1 & ?
\end{array}\right] \quad \text { where } \beta=\left[\begin{array}{lll}
\beta_{1} & ? \\
0 & & \beta_{n}
\end{array}\right]
$$

is an upper triangular matrix. Now consider the adjoint action of $\mathfrak{h}$ on the basis vectors $w_{1}, \ldots, w_{m}$. For $H \in \mathfrak{h}, \operatorname{ad}(H)\left(w_{j}\right)=p\left(\left[H, w_{j}^{\prime}\right]\right)$ where $p: \mathfrak{g} \rightarrow \mathfrak{g} / \mathfrak{h}$ and $p\left(w_{j}^{\prime}\right)$ $=w_{j}$. Since $\left[H, w_{j}^{\prime}\right] \in \mathfrak{n}, p\left(\left[H, w_{j}^{\prime}\right]\right) \in \mathfrak{n} / \mathfrak{h}^{\prime}$ and thus may be written as a linear combination of $v_{1}, \ldots, v_{n}$. Therefore

$$
\theta={ }_{w}^{v}\left[\begin{array}{ccc}
v & w \\
\beta & \frac{1}{2} & ? \\
0 & 1 & 0
\end{array}\right]
$$

where $\beta$ is upper triangular with diagonal entries $\beta_{1}, \ldots, \beta_{n}$.

It will be proved that the $\tau_{j}$ 's are zero by showing that the curvature matrix $\Omega=d^{\prime \prime} \theta$ is strictly upper triangular; that is, $d \beta_{j}=0$. Now $d \beta_{j}=0$ if and only if $\beta_{j}$ vanishes on all brackets. Since $\beta_{j} \in \mathfrak{h}^{*}$, this is equivalent to showing that $\beta_{j}$ vanishes on $\mathfrak{h}^{\prime}=\mathfrak{h} \cap \mathfrak{n}$. But this is clear because $\mathfrak{h}^{\prime} \subset \mathfrak{n}$ and thus acts nilpotently on $\mathfrak{g} / \mathfrak{h}$. Therefore the entries of $\beta$ which record this action must be strictly upper triangular. The diagonal (semisimple) entries $\beta_{j}$ must vanish on $\mathfrak{h}^{\prime}$. 
Therefore the connection matrix $\theta$ and the curvature matrix $\Omega$ have the following forms:

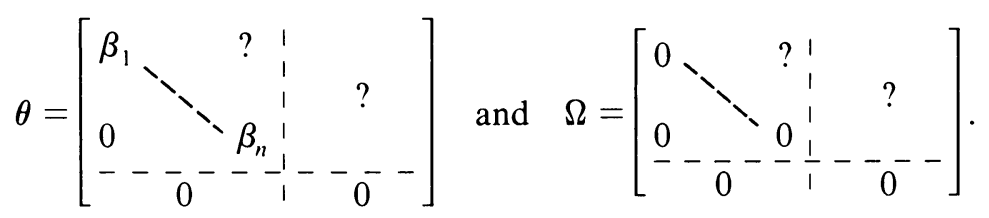

Then $\tau_{j}=\operatorname{Tr}\left(\Omega^{j}\right)=0$ for all $j . h_{i}=\int_{0}^{1} \operatorname{Tr}\left(\Omega_{t}^{i}\right)$ (integral of the " $d t$-terms") where $\Omega_{t}=t \Omega+\left(t-t^{2}\right) \theta^{2}+\theta d t$. Since the diagonal entries of $\theta$ are differential 1-forms, it is clear from the above forms for $\theta$ and $\Omega$ that

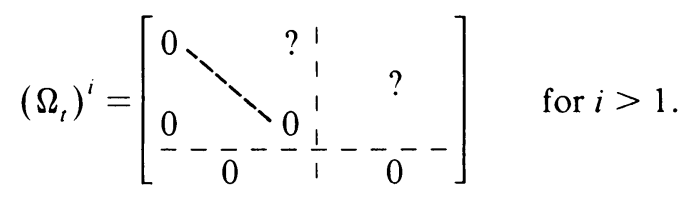

Thus $h_{i}=0$ for $i>1$.

THEOREM 4. Let $\mathfrak{g}$ be a semisimple Lie algebra with $\mathfrak{h} \subset \mathrm{g}$ a solvable subalgebra of codimension $q$. If $\mathfrak{h}$ is not a Borel subalgebra of $\mathfrak{g}$, then the foliation classes for $(\mathfrak{g}, \mathfrak{h})$ vanish.

Proof. Let $V=\mathfrak{g} / \mathfrak{h}$. The special connection matrix $\theta$ records the adjoint action $\mathfrak{h} \times V \rightarrow V$. h is solvable, so by Lie's theorem there exists a basis of $V$ such that $\theta$ is upper triangular. Thus the foliation classes are determined by the diagonal entries $\theta_{k k}$, hereafter denoted $\theta_{k}$.

In the case of a solvable subalgebra it is known (Pittie [6]) that the only possible nonzero secondary forms are of the type $h_{i} \tau_{J}$ where $h_{i}=\sum_{k=1}^{q} \theta_{k}\left(d \theta_{k}\right)^{i-1}, \tau_{j}=$ $\sum_{k=1}^{q}\left(d \theta_{k}\right)^{j}$, and $i+|J|=q+1$. Hence any form of the type $h_{i} \tau_{J}$ will be a sum of terms with each term having a $q$-fold product of various $d \theta_{k}$ 's as a factor. The root space decomposition of $g$ with respect to a Borel subalgebra $\mathfrak{b} \supset \mathfrak{h}$ will be used to show that if $q$ is greater than the number of positive roots (which is implied by $h \neq$ Borel subalg), then $d \theta_{k_{1}} \wedge \cdots \wedge d \theta_{k_{q}}=0$; hence $h_{i} \tau_{J}=0$.

Since $\mathfrak{h}$ is solvable, there is a Borel subalgebra $\mathfrak{b}$ containing $\mathfrak{h}$. Choose a maximal toral (Cartan) subalgebra $t \subset \mathfrak{b}$ and consider the root space decomposition of $g$ with respect to $t$ :

$$
\mathfrak{g}=\mathrm{t}+\sum g_{\alpha}+\sum g_{-\alpha}=\mathfrak{b}+\sum g_{-\alpha} \quad\left(0<\alpha \in \mathrm{t}^{*}\right)
$$

Now I claim that each $\theta_{k} \in \mathrm{t}^{*}$. For let $X \in \mathfrak{h}$ and write $X=X_{\mathrm{t}}+X_{\mathfrak{n}}$ where $X_{\mathrm{t}} \in \mathrm{t}$ and $X_{\mathrm{n}} \in \Sigma_{0<\alpha} g_{\alpha} . X_{\mathrm{n}}$ acts nilpotently on $V$ (since $X_{\mathrm{n}}$ can be written as a sum of vectors in positive root spaces) and $X_{\mathrm{t}}$ acts semisimply. $\left(\theta_{i j}(X)\right)$ is the matrix for $\operatorname{ad}(X): V \rightarrow V$, and the diagonal $\left(\theta_{k}(X)\right)$ is the semisimple part of $\operatorname{ad}(X)$. Hence $\theta_{k} \in \mathrm{t}^{*}$. 
Now since $\theta_{k} \in \mathrm{t}^{*}$.

$$
d \theta_{k}=-\sum \theta_{k}\left(H_{\alpha}\right) \xi_{\alpha} \wedge \xi_{-\alpha} \quad\left(0<\alpha \in \mathrm{t}^{*}\right),
$$

where $\left\{X_{\alpha}, X_{-\alpha}, H_{\alpha}\right\}_{\alpha \in t^{*}}$ are Weyl generators for $\mathfrak{g}$ and $\xi_{\alpha}, \xi_{-\alpha}$ are dual to $X_{\alpha} \in \mathfrak{g}_{\alpha}$, $X_{-\alpha} \in \mathrm{g}_{-\alpha}$. So if $q$ is greater than the number of positive roots, $d \theta_{k_{1}} \wedge \cdots \wedge d \theta_{k_{4}}$ involves some $\xi_{\alpha}$ twice and hence is zero. Thus $h_{i} \tau_{J}=0$.

It remains only to remark that $\mathfrak{h}$ is properly contained in $\mathfrak{b}$ if and only if $q=\operatorname{codim} \mathfrak{h}>\operatorname{codim} \mathfrak{b}=$ number of positive roots.

THEOREM 5. Let $(\mathfrak{g}, \mathfrak{h})$ be a homogeneous foliation of codimension $q$ with $\mathfrak{h}$ solvable. Then the foliation classes for $(\mathfrak{g}, \mathfrak{h})$ all vanish except in the case in which $\mathfrak{h}^{\prime \prime}$ is a Borel subalgebra of $\mathfrak{g}(\mathfrak{g})$ and $\Re(g) \subseteq \mathfrak{h}$; in this case the only possible nonzero class is $h_{1} \tau_{1}^{q}$.

Proof. As in the proof of the Addition Theorem, the Levi decomposition $\mathfrak{g} \approx \mathfrak{g}(\mathfrak{g}) \ltimes \mathcal{R}(\mathfrak{g})$ induces a (solvable, solvable) foliation $\left(\mathscr{R}(\mathfrak{g}), \mathfrak{h}^{\prime}\right)$ and a (semisimple, solvable) foliation $\left(\mathfrak{g}(\mathfrak{g}), \mathfrak{h}^{\prime \prime}\right)$. By the Addition Theorem and Theorem 3, $h_{i}=h_{i}(\Re)+h_{i}(\mathfrak{g})$ and $\tau_{i}=\tau_{i}(\Re)+\tau_{i}(\mathfrak{g})=\tau_{i}(\mathfrak{g})$.

Since $\mathfrak{h}$ is solvable, the foliation classes are represented by $h_{i} \tau_{J}$ where $i+|J|=q$ +1 (Pittie [6]). Since there is only one $h_{i}$ factor, it is not necessary to use the "integration over the fibre" definition to calculate $h_{i}$. Any form $g_{i} \tau_{J}$ such that $d g_{i}=\tau_{i}$ will give the same cohomology class as $h_{i} \tau_{J}$. To verify this, it suffices to show that $h_{i} \tau_{J}-g_{i} \tau_{J}$ is exact. Now $\tau_{J}$ is exact since by construction each $\tau_{i}=d h_{i}$; so write $\tau_{J}=d f$. Then

$$
d\left(\left(g_{i}-h_{i}\right) f\right)=d\left(g_{i}-h_{i}\right) f-\left(g_{i}-h_{i}\right) d f=\left(h_{i}-g_{i}\right) \tau_{J} .
$$

Thus for the purpose of calculating the foliation cohomology classes for $(\mathfrak{g}, \mathfrak{h}), \mathrm{I}$ may select $h_{i}(\Re)=0$ since $\tau_{i}(\Re)=0$. So the foliation classes for $(\mathfrak{g}, \mathfrak{h})$ are constructed from the forms $h_{i}=h_{i}(\mathfrak{B})$ and $\tau_{i}=\tau_{i}(\mathfrak{g})$.

The only nonzero classes for $(\mathfrak{g}, \mathfrak{h})$ are those combinations of $h$ and $\tau$ forms which also give nonzero classes for $\left(\mathfrak{g}, \mathfrak{h}^{\prime \prime}\right)$. This is because the exterior derivatives for $\mathfrak{g}$ and for $\mathfrak{g} \subset \mathfrak{g}$ agree on $\Lambda^{*} \mathfrak{g}^{*}$. Thus if a secondary form is exact for $\left(\mathfrak{I}, \mathfrak{h}^{\prime \prime}\right)$, then it is also exact for $(g, \mathfrak{h})$.

By Theorem 4 , the foliation classes for $\left(\mathfrak{g}, \mathfrak{h}^{\prime \prime}\right)$ and hence for $(\mathfrak{g}, \mathfrak{h})$ are all zero except when $\mathfrak{h}^{\prime \prime}$ is a Borel in $\mathfrak{g}$. Then for $\left(\mathfrak{g}, \mathfrak{h}^{\prime \prime}\right)$ the only class is $h_{1} \tau_{1}^{p}$ where $p=\operatorname{codim}\left(\mathfrak{g}, \mathfrak{h}^{\prime \prime}\right)$ (Pittie [6]). Thus the only possible class for $(\mathfrak{g}, \mathfrak{h})$ is $h_{1} \tau_{1}^{q}$ where $q=p(i+|J|=q+1)$. If $\operatorname{codim}\left(\Re, \mathfrak{h}^{\prime}\right)>0$, then $q>p$ and the classes all vanish. If $\Re(g) \subset \mathfrak{h}$, then $q=p$ and $h_{1} \tau_{1}^{q}$ is the only foliation class.

In closing we remark that Roussaire's example of $(S l(2, \mathbf{R}), B)$ where $B$ is the subgroup of upper triangular matrices is an example where the class $h_{1} \tau_{1}(q=1)$ of Theorem 5 is nonzero for the induced foliation of $\Gamma \backslash S l(2, \mathbf{R})$ where $\Gamma$ is a discrete subgroup of $S l(2, \mathbf{R})$ with compact quotient.

\section{REFERENCES}

1. D. Baker, On a class of foliations and evaluation of their characteristic classes, Comment. Math. Helv. 53 (1978).

2. R. Bott, On the characteristic classes of groups of diffeomorphisms, Enseign. Math. (2) 23 (1977). 
3. D. Fuks, Finite dimensional Lie algebras of formal vector fields and secondary classes of foliations, Uspehi Mat. Nauk 40 (1) (1976).

4. C. Godbillon, Cohomologies d'algèbres de Lie de champs de vecteurs formels, Séminaire Bourbaki (1972-73), Exposé 421, Lecture Notes in Math., vol. 383, Springer-Verlag, New York, 1974.

5. F. Kamber and P. Tondeur, Non-trivial characteristic invariants of homogeneous foliated bundles, Ann. Sci. École Norm. Sup. (4) 8 (1975), 433-486.

6. H. Pittie, The secondary characteristic classes of parabolic foliations, Comment. Math. Helv. 54 (1979), 601-614.

Department of Mathematics, Wayne State University, Detroit, Michigan 48202 